\title{
A Low-Power 512-Bit EEPROM Design for UHF RFID Tag Chips
}

\author{
Jae-Hyung Lee ${ }^{1}$, Gyu-Ho Lim ${ }^{1}$, Ji-Hong Kim ${ }^{1}$, Mu-Hun Park ${ }^{1}$, Kyo-Hong Jin ${ }^{1}$, \\ Jeong-won $\mathrm{Cha}^{1}$, Pan-Bong $\mathrm{Ha}^{1}$, Yung-Jin Gang ${ }^{2}$, and Young-Hee Kim ${ }^{1}$ \\ ${ }^{1}$ Changwon National University, \\ 9 Sarim-dong, Changwon, Gyeongnam, 641-773, Korea \\ \{tommo, ghlim\}@changwon.ac.kr, lapael81@empal.com, \\ mhparkesarim. changwon.ac.kr \\ $\{k h j i n, j$ cha, pha, youngkim\} @ changwon. ac.kr \\ ${ }^{2}$ DavitDyne Co., Ltd. \\ B 901-3, Ssangyoung IT Twin Tower, Sangdaewon-dong, \\ Sungnam, Kyungki, 462-723, Korea \\ jim@davitdyne.com
}

\begin{abstract}
In this paper, a design for a low-power 512-bit synchronous EEPROM with flash cells for passive UHF RFID tag chip is presented. Applied are low-power schemes such as dual power supply voltage $(\mathrm{VDD}=1.5 \mathrm{~V}$ and $\mathrm{VDDP}=2.5 \mathrm{~V}$ ), clocked inverter sensing, voltage-up converter, IO interface, and Dickson charge pump using schottky diode. An EEPROM is fabricated with the $0.25 \mu \mathrm{m}$ EEPROM process. Simulation results show that power dissipations are $8.34 \mu \mathrm{W}$ in the read cycle and $57.7 \mu \mathrm{W}$ in the write cycle, respectively. The layout size is $449.3 \mu \mathrm{m} \times 480.67 \mu \mathrm{m}$.
\end{abstract}

Keywords: Low-Power, EEPROM, UHF RFID, Tag, Charge pump.

\section{Introduction}

RFID(Radio Frequency IDentification) is the technology to provide various services communicating between things by collecting, storing, and revising the information around these things through they installed RFID tags on item. RFID tags are classified according to communication method, battery existence, and read/write[1]. They are standardized by EPC global which sets EPC (Electronic Product Code). Currently, Generation 2 of Class 1 is one of the widely selected standards. It has advantages in terms of cost and area. This paper presented EEPROM design for a passive UHF RFID tag chip.

\section{Circuit Design}

Fig. 1 is a block diagram of a 512-bit synchronous EEPROM. The memory cell array is composed of flash cell[8]. The EEPROM has four operating modes : program, erase, read, and stand-by. It is synchronously operated by clock. Write mode means 
program and erase modes. Dual power supply voltage, $\operatorname{VDD}(1.5 \mathrm{~V})$ and $\operatorname{VDDP}(2.5 \mathrm{~V})$, is used to reduce currents in read and write modes.

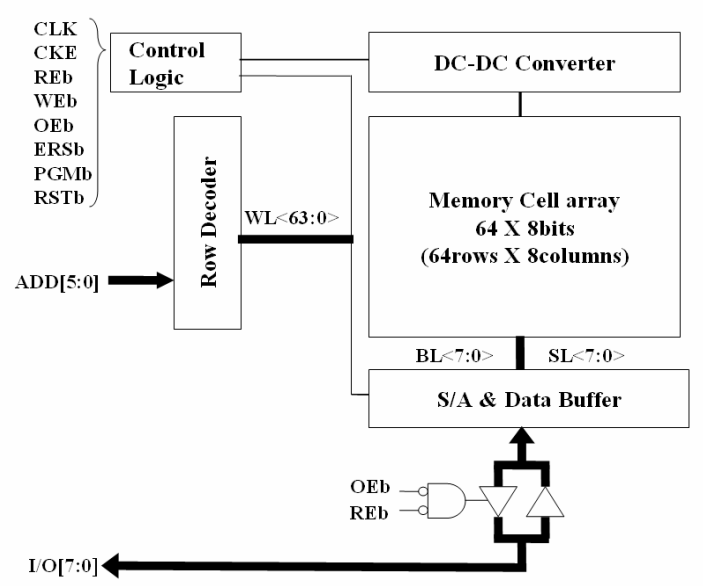

Fig. 1. Block diagram of 512-bit synchronous EEPROM

A clocked inverter sensing method[6] is applied to read data of EEPROM cell in the read mode. And a current sensing circuit is used for the non-volatile memory[4]. This is not proper in the EEPROM design for RFID tag chips because the current dissipation of the sensing circuit is large. Therefore, despite low speed, low-power $\mathrm{RD}($ Read Data) sense amplifier is used without a reference current biasing circuit.

DC-DC converter uses Dickson charge pump[5] to generate high voltage in the write mode. VDDP power is used in the voltage-up converter, VPP control logic, and charge pump circuits[8]. VREF_VPP is reference voltage required for the DC-DC converter. VREF_VPP level is too high to generate it by using VDD, so a low-power voltage-up converter is added to the DC-DC converter. The voltage-up converter makes reference voltage double by using VDDP.

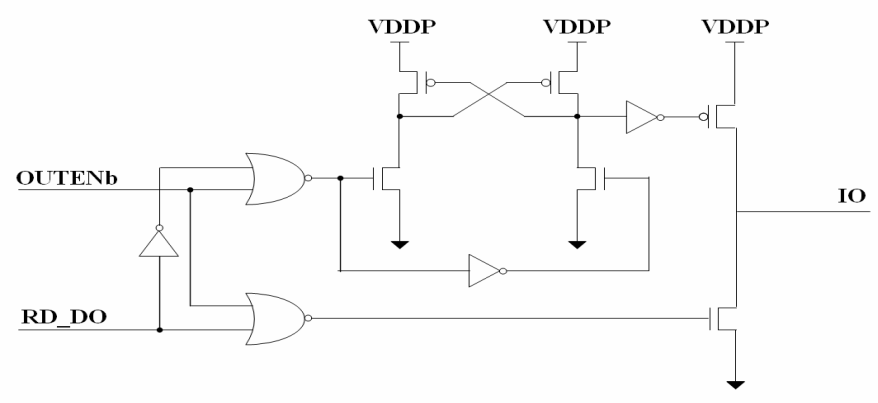

Fig. 2. IO Interface circuit 
The voltage of RD_DO swings between VDD and VSS. The voltage of IO swings between VDDP and VSS. If VDD voltage transfers to the IO, they induce short circuit currents in other IO interface. So, level translator is newly applied to the IO interface.

Dickson charge pump generates high voltages, VPP and VPPL, in the write mode. The lower the forward bias voltage of the diode is used, the lower current flows in Dickson charge pump. For this reason, a schottky diode is used for the pump. Power dissipations of the charge pump are $67.7 \mu \mathrm{W}$ with PN diode and $57.7 \mu \mathrm{W}$ with schottky diode in the program mode. Approximately, the power dissipation with schottky diode drops by $12 \%$ compared with PN diode.

\section{Simulation Results}

Fig. 3 shows timing diagrams of CLK signal from analog circuit, command control signals, CKE, REb, OEb from logic circuit, and PRECHARGE, DLINE_LOADb and $\mathrm{SAENb}$ from control logic circuit as shown in Fig. 1. When read command enters at a rising edge, PCHARGE makes DLINE and BL precharge to VDD. WL is active after $\mathrm{BL}$ is precharged. When a data is transferred to the $\mathrm{BL}$, a valid data comes out of $\mathrm{I} / \mathrm{O}$ through the RD_DO within a half clock period with SAENb activated. Power dissipation simulation results are $57.7 \mu \mathrm{W}$ in the program mode, $42.3 \mu \mathrm{W}$ in the erase mode, and $8.34 \mu \mathrm{W}$ in the read mode, respectively. Fig. 4 is a layout picture with $0.25 \mu \mathrm{m}$ EEPROM process. Layout size is $449.3 \mu \mathrm{m} \times 480.67 \mu \mathrm{m}$.

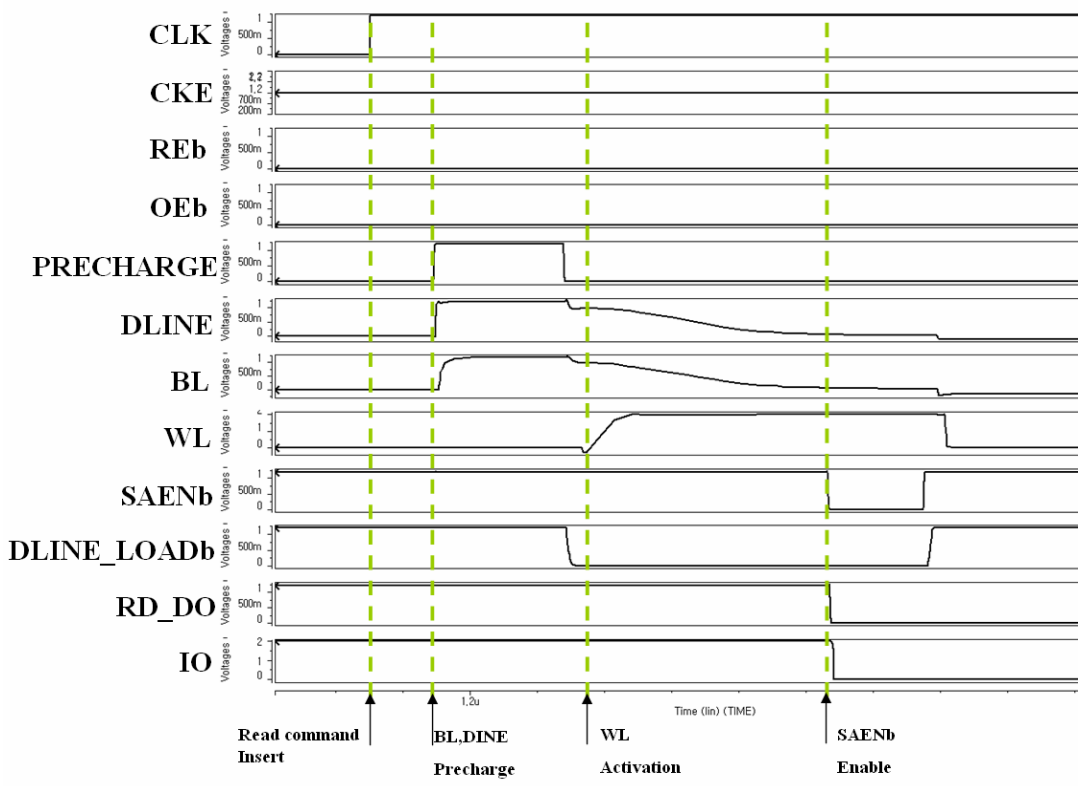

Fig. 3. Simulation result for the case of critical path in the read cycle 


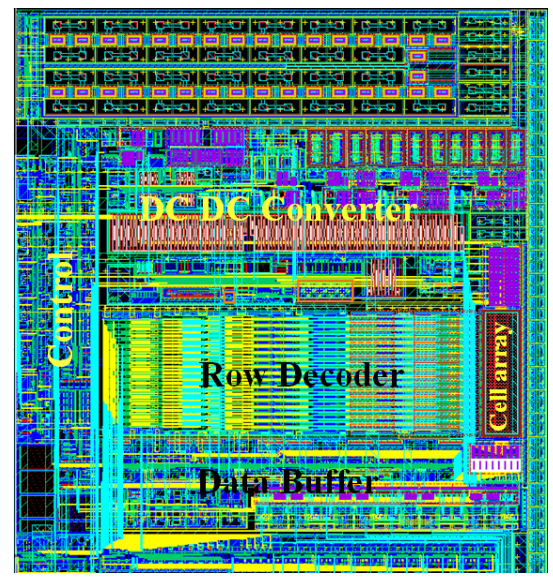

Fig. 4. EEPROM layout picture

\section{Conclusions}

The EEPROM is fabricated with the 0.25 EEPROM process. In this paper, to reduce power dissipation in EEPROM, dual power supply voltage, VDD $(1.5 \mathrm{~V})$ and $\operatorname{VDDP}(2.5 \mathrm{~V})$, is used to reduce the currents in the read and write modes. Also sensing method using clocked inverter in the read mode is applied. VREF_VPP is made by using the voltage-up converter in the write cycle. Level translator is newly applied to IO interface in order to reduce short circuit current. A schottky diode is used for lower power dissipation in Dickson charge pump. Simulation result shows that the designed EEPROM is suitable for UHF RFID Class 1 Generation 2 and the fabricated EEPROM will be verified by measurement in near future.

Acknowledgments. This work is supported by IT R\&D Project funded by Korean Ministry of Information and Communications.

\section{References}

1. http://www.epcglobalinc.org

2. Weinstein, R.: RFID: a technical overview and its application to the enterprise. IT Professional, vol.7, Issue 3. (2005) 27-33

3. Junghwan Lee and Minkyung Ko: A novel EEPROM cell for smart card application. Microelectronic Engineering, vol.71, Issues 3-4. (2004) 283-287

4. Fei Xu, Xiangqing He, and Li Zhang: Key Design Techniques of a 40ns 16K Bits Embedded EEPROM Memory. ICCCAS 2004, vol. 2. (2004) 1516-1520

5. J.F.Dickson: On-Chip High-Voltage Generation in MNOS Integrated Circuits Using an Improved Voltage Multiplier Technique. IEEE JSSC, vol. 11. (1976) 374-378

6. YoungHee Kim et al.:A low-power EEPROM design for UHF RFID tag chip. Journal of KIMICS, vol.10, No.3 (2006) 486-495 